\title{
Numerical Model for the Orbit of the Earth
}

\author{
S. Karna ${ }^{1, *}$, A. K. Mallik ${ }^{2}$ \\ ${ }^{1}$ Physics Department, Tri-Chandra M. Campus, Tribhuvan University, Nepal \\ ${ }^{2}$ Physics Department, Tribhuvan M. Campus, Tribhuvan University, Nepal
}

Copyright $\bigcirc 2017$ by authors, all rights reserved. Authors agree that this article remains permanently open access under the terms of the Creative Commons Attribution License 4.0 International License

\begin{abstract}
In this paper, we constructed a 3-D numerical model of the Earth-Sun geometry. Our model defines Earth's orbit as an inclined plane of spherically symmetric system. We calculated the degree of the tilt of the Earth orbit to the ecliptic plane by converting from ecliptic frame of reference to the orbital frame of reference and then we made all the measurement. Initial inputs of our model are aphelion and perihelion parameters. It is interesting to examine that our results obtained from Earth inclined orbit are same that observed value from Earth's circular orbit. In other words, values of the axial tilt of Earth and Sun, the time taken for the Sun to move from vernal equinox to autumnal equinox and then back to the vernal equinox does not change. Moreover, we were also able to derive mathematical relations for finding the length of the apparent solar days throughout the year. On introducing the new types of the length of the day, called Saurya day, the rate of precession of equinox is calculated.
\end{abstract}

Keywords Orbit of the Earth, Earth-Sun Geometry, Equinox, Perihelion, Aphelion, Rate of Precession

\section{Introduction}

The Solar system consists of the Sun, planets, satellites of the planets, numerous comets, asteroids, meteoroids and the interplanetary medium. Planets and most of their (planet's) satellites revolve in a nearly circular orbit around the Sun and at the same time, they rotate about their own axis. Earth revolves around the sun in an anti-clockwise direction and always points into space in the same direction. During the revolution period of the Earth around the Sun, the distance between the Earth and the Sun is not always the same, i.e., the orbit of Earth deviates a bit from the circle. The aphelion (farthest) distance of the Earth is $1.016710 \mathrm{AU}$, and the perihelion (closest) distance is $0.983290 \mathrm{AU}$, where $1 \mathrm{AU}=$ $149,597,870.7 \mathrm{~km}$. The mean distance between the Earth and Sun is $1 \mathrm{AU}[1]$.

In our solar system, plane of the Earth's orbit is known as the ecliptic plane. The ecliptic coordinate system is used as the main reference for specifying the position of the other celestial objects in our solar system. The perihelion and aphelion distances are said to be lie on the same ecliptic plane, though the shape of the Earth's orbit is not circular. Since the circular shape appears elliptical when viewed at an angle, means that for elliptical shape of orbit of Earth around the Sun, the plane in which Earth orbits must be inclined to some angle with the ecliptic plane. In another word, the plane of the Earth's orbit must be in orbital plane, other than the ecliptic plane.

The article is organized as follow: In Section 3, we describe our 3-D numerical model of the Earth-Sun geometry. In Section 4, we present the results: values of the axial tilt of Earth and Sun, the time taken for the Sun to move from vernal equinox to autumnal equinox and then back to the vernal equinox. In Section 5, we discuss what we are able to draw from our 3-D numerical model and compare our results with existing theory. In Section 6, we summarize our results and present our conclusions.

\section{Numerical Model}

The angle of cosine of ratios of mean distance to aphelion distance $\left(\theta_{1}\right)$ and perihelion distance to mean distance $\left(\theta_{2}\right)$ are as follows:

$$
\begin{aligned}
\operatorname{Cos}^{-1}(1 / 1.016710)= & \operatorname{Cos} 10.402169^{\circ}=\operatorname{Cos} \theta_{1} \Rightarrow \theta_{1}= \\
& 10.402169^{\circ}
\end{aligned}
$$

and $\operatorname{Cos}^{-1}(0.983290 / 1)=\operatorname{Cos} 10.488961^{\circ}=\operatorname{Cos} \theta_{2} \Rightarrow \theta_{2}=$ $10.488961^{\circ}$

or, Mean value, $\theta=10.445565^{\circ}$

We took the average value of $\theta_{1}$ and $\theta_{2}$ for our further calculation.

\subsection{To Find the Inclination of the Earth's Orbit}

For Earth-Sun Geometry only,

Consider a fictitious Celestial sphere of radius $\mathrm{R}_{\mathrm{c}}(=$ $\left.149,597,870.7 \mathrm{~km} / \operatorname{Sin} 10.445565^{\circ}\right)=825,134,101.1 \mathrm{~km}$ centered on the Sun, with its equator running along the plane of the ecliptic as shown in fig. 2. Normal to the ecliptic plane 
defines the ecliptic pole of the Solar System. This Celestial sphere is rotating uniformly about pole to the ecliptic, so the reference frame fixed on it is an ecliptic frame of reference $\mathrm{E}_{\mathrm{S}}$.

Let us consider another frame of reference $\mathrm{O}_{\mathrm{s}}$, called the orbital frame of reference with its origin coincides with $E_{S}$ but its coordinate axes inclined at an angle of $\lambda$ as shown in fig. 1. (This figure is adapted from Mathur (1996) [2]).

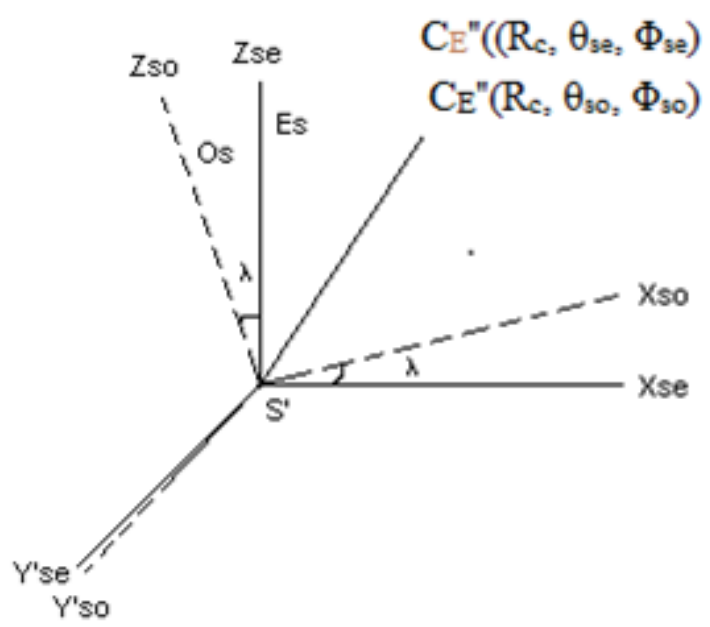

Figure 1. Representation of the point $\mathrm{C}_{\mathrm{E}}$ " in ecliptic and orbital frame of reference

Let any point $\mathrm{C}_{\mathrm{E}}$ " lies at the co-latitude of $10.445565^{\circ}$ on the surface of the Celestial sphere, and from $\mathrm{C}_{\mathrm{E}}{ }^{\prime \prime}, \mathrm{C}_{\mathrm{E}}{ }^{\prime \prime} \mathrm{C}_{\mathrm{E}}{ }^{\prime}$ perpendicular to $\mathrm{x}_{\mathrm{se}} \mathrm{y}_{\mathrm{se}^{-}}$plane is drawn, then the radial distance from the center of the celestial sphere $\left(\mathrm{S}^{\prime}\right)$ in its celestial equatorial plane $\left(\mathrm{S}^{\prime} \mathrm{C}_{\mathrm{E}}{ }^{\prime}\right)$ is

$$
\mathrm{R}_{10.445565^{\circ}}{ }^{\circ}=\mathrm{R}_{\mathrm{c}} \operatorname{Sin} \theta=149,597,870.7 \mathrm{~km} .
$$

This Celestial Sphere rotates uniformly, then the point $\mathrm{C}_{\mathrm{E}}{ }^{\prime}$ rotates on the equatorial plane (or the ecliptic plane also) of the celestial sphere $\left(\mathrm{z}_{\mathrm{se}}=0\right)$ uniformly at the distance of $149,597,870.7 \mathrm{~km}$. from the center. Here $\mathrm{C}_{\mathrm{E}}^{\prime}$ is taken as the imaginary center of the Earth of radius $6371 \mathrm{~km}$., with its equator running along the equator of the Celestial Sphere, and the path followed by $\mathrm{C}_{\mathrm{E}}^{\prime}$ in equatorial plane is the imaginary orbit of the Earth around the Sun.

Again, from $\mathrm{C}_{\mathrm{E}}{ }^{\prime \prime}, \mathrm{C}_{\mathrm{E}}{ } \mathrm{C}_{\mathrm{E}}$ perpendicular to $\mathrm{x}_{\mathrm{so}} \mathrm{y}_{\mathrm{so}}-$ plane is drawn. Then, the radial distance from the center of the Celestial sphere in orbital plane is $S^{\prime} C_{E}$. The path of $C_{E}$ in orbital plane is not circular, but deviates a bit from the circle. Thus the projection of this point $\mathrm{C}_{\mathrm{E}}$ " (lying on the Celestial sphere) in orbital plane as $C_{\mathrm{E}}$, (center of Earth, $\mathrm{z}_{\mathrm{so}}=0$ ) defines the actual orbit of the Earth around the Sun. The orbital plane is inclined at an angle $\lambda$ with the ecliptic plane and the value of $\lambda$ represents the tilt of the Earth's orbit to the ecliptic.

\subsection{Calculating $\lambda$ (Tilt of the Earth's Orbit to the Ecliptic Plane)}

Let the Cartesian Coordinates of the point $\mathrm{C}_{\mathrm{E}}$ " are $\mathrm{x}_{\mathrm{se}}, \mathrm{y}_{\mathrm{se}}$, $z_{\mathrm{se}}$ in frame $\mathrm{E}_{\mathrm{S}}$ and $\mathrm{x}_{\mathrm{so}}, \mathrm{y}_{\mathrm{so}}, \mathrm{z}_{\mathrm{so}}$ in frame $\mathrm{O}_{\mathrm{s}}$. Then the transformation equations from $E_{S}$ to $O_{S}$ is

$$
\begin{gathered}
\mathrm{x}_{\mathrm{so}}=\mathrm{x}_{\mathrm{se}} \cos \lambda+\mathrm{z}_{\mathrm{se}} \sin \lambda \\
\mathrm{y}_{\mathrm{so}}=\mathrm{y}_{\mathrm{se}} \\
\mathrm{z}_{\mathrm{so}}=-\mathrm{x}_{\mathrm{se}} \sin \lambda+\mathrm{z}_{\mathrm{se}} \cos \lambda
\end{gathered}
$$

Let the spherical polar coordinates of the point $\mathrm{C}_{\mathrm{E}}$ " are specified by $\left(\mathrm{R}_{\mathrm{c}}, \theta_{\mathrm{se}}, \Phi_{\mathrm{se}}\right)$ with respect to the axes $\mathrm{x}_{\mathrm{se}}, \mathrm{y}_{\mathrm{se}}, \mathrm{z}_{\mathrm{se}}$ in frame $E_{S}$ and $C_{E} "\left(R_{c}, \theta_{s o}, \Phi_{s o}\right)$ with respect to $x_{s o}, y_{s o}, z_{s o}$ in frame $\mathrm{O}_{\mathrm{s}}$. Here, $\theta_{\mathrm{se}}$ and $\theta_{\mathrm{so}}$ is the co-latitude i.e. the angle between $\mathrm{C}_{\mathrm{E}}{ }^{\prime \prime} \mathrm{S}^{\prime}$ and $\mathrm{z}_{\mathrm{se}^{-}}$axis in frame $\mathrm{E}_{\mathrm{S}}$ and $\mathrm{C}_{\mathrm{E}}{ }^{\prime \prime} \mathrm{S}^{\prime}$ and $\mathrm{z}_{\mathrm{so}}$-axis in frame $\mathrm{O}_{\mathrm{S}}$ respectively. $\Phi_{\mathrm{se}}$ and $\Phi_{\mathrm{so}}$ is the longitudinal or azimuthal angle.

The longitudinal or azimuthal angle $\left(\Phi_{\mathrm{se}}\right)$ is measured by starting the revolution of the Earth from the point, where the Earth is at a farthest distance to the Sun.

Substituting the value of $\mathrm{x}_{\mathrm{se}}$ for aphelion distance and perihelion distance in the transformation equation and solving it, we get, $\lambda=0.176506$ degree.

Here, the value of $\lambda$ represents the degree of tilt of the Earth's orbit to the ecliptic plane. Thus the deviation in the path of the Earth from the circle indicates that the Earth's orbital plane is inclined at an angle of 0.176506 degree with the ecliptic plane.

\subsection{Distance between Earth and Sun in Orbital Plane}

For the given value of $\mathrm{R}_{\mathrm{c}}=825,134,101.1 \mathrm{~km} ., \theta_{\text {se }}$ $=10.445565^{\circ}, \mathrm{z}_{\mathrm{se}}=\mathrm{R}_{\mathrm{c}} \cos \theta_{\mathrm{se}}=811,459,649 \mathrm{~km} ., \lambda=$ $0.176506^{\circ}$ the value of $\mathrm{x}_{\mathrm{se}}, \mathrm{y}_{\mathrm{se}}, \mathrm{z}_{\mathrm{se}}, \mathrm{x}_{\mathrm{so}}, \mathrm{z}_{\mathrm{so}}$, and $\mathrm{R}_{\mathrm{so}}$ (the distance between Earth and Sun in orbital plane) can be calculated as follows in Table: 1 ; 
Table1. Distance between Earth and Sun in orbital plane at different azimuthal angle $\left(\Phi_{\mathrm{se}}\right)$.

\begin{tabular}{|c|c|c|c|c|c|}
\hline$\Phi_{\mathrm{se}}$ & $\mathrm{x}_{\mathrm{se}}=\mathrm{R} \sin \theta_{\mathrm{se}} \cos \Phi_{\mathrm{se}}$ & $\mathrm{y}_{\mathrm{se}}=\mathrm{R} \sin \theta_{\mathrm{se}} \sin \Phi_{\mathrm{se}}=\mathrm{y}_{\mathrm{so}}$ & $\mathrm{x}_{\mathrm{so}}=\mathrm{x}_{\mathrm{se}} \cos \lambda+\mathrm{z}_{\mathrm{se}} \sin \lambda$ & $\mathrm{z}_{\mathrm{so}}=-\mathrm{x}_{\mathrm{se}} \sin \lambda+\mathrm{z}_{\mathrm{se}} \cos \lambda$ & $\mathrm{R}_{\mathrm{so}}=\left(\mathrm{x}_{\mathrm{so}}{ }^{2}+\mathrm{y}_{\mathrm{so}}{ }^{2}\right)^{1 / 2}$ \\
\hline $0^{\circ}$ & $149,597,870.7$ & 0 & $152,096,948.3$ & $810,994,946.5$ & $152,096,948.3$ \\
\hline $30^{\circ}$ & $129,555,556.4$ & $74,798,935.35$ & $132,054,729.1$ & $811,056,688.9$ & $151,767,362.1$ \\
\hline $60^{\circ}$ & $74,798,935.35$ & $129,555,556.4$ & $77,298,367.9$ & $811,225,372.5$ & $150,863,116.3$ \\
\hline $90^{\circ}$ & 0 & $149,597,870.7$ & $2,499,787.4$ & $811,455,798.6$ & $149,618,755.0$ \\
\hline $120^{\circ}$ & $-74,798,935.35$ & $129,555,556.4$ & $-72,298,793.0$ & $811,686,224.6$ & $148,363,599.5$ \\
\hline $150^{\circ}$ & $-129,555,556.4$ & $74,798,935.35$ & $-127,055,154.2$ & $811,854,908.2$ & $147,437,759.5$ \\
\hline $180^{\circ}$ & $-149,597,870.7$ & 0 & $-147,097,373.4$ & $811,916,650.7$ & $147,097,373.4$ \\
\hline $210^{\circ}$ & $-129,555,556.4$ & $-74,798,935.35$ & $-127,055,154.2$ & $811,854,908.2$ & $147,437,759.5$ \\
\hline $240^{\circ}$ & $-74,798,935.35$ & $-129,555,556.4$ & $-72,298,793.0$ & $811,686,224.6$ & $148,363,599.5$ \\
\hline $270^{\circ}$ & 0 & $-149,597,870.7$ & $2,499,787.4$ & $811,455,798.6$ & $149,618,755.0$ \\
\hline $300^{\circ}$ & $74,798,935.35$ & $-129,555,556.4$ & $77,298,367.9$ & $811,225,372.5$ & $150,863,116.3$ \\
\hline $330^{\circ}$ & $129,555,556.4$ & $-74,798,935.35$ & $132,054,729.1$ & $811,056,688.9$ & $151,767,362.1$ \\
\hline $360^{\circ}$ & $149,597,870.7$ & 0 & $152,096,948.3$ & $810,994,946.5$ & $152,096,948.3$ \\
\hline
\end{tabular}

The distance between the Earth and the Sun $\left(\mathrm{R}_{\mathrm{so}}\right)$ at any instant in orbital plane is obtained by using the formula, $\mathrm{R}_{\mathrm{so}}=$ $\left(\mathrm{x}_{\mathrm{so}}{ }^{2}+\mathrm{y}_{\mathrm{so}}{ }^{2}\right)^{1 / 2}$ in $360^{\circ}$ revolution period. Here, $360^{\circ}$ period of revolution indicates the total number of days 365.242190 in one year.

\subsection{Shift of the Center of the Fictitious Celestial Sphere in Ecliptic Frame}

To get the value of $x_{\text {so }}=0$, we have

$$
\mathrm{x}_{\mathrm{so}}=\mathrm{x}_{\mathrm{se}} \cos \lambda+\mathrm{z}_{\mathrm{se}} \sin \lambda=0
$$

or, $\mathrm{x}_{\mathrm{se}}=\mathrm{R}_{\mathrm{c}} \sin \theta_{\mathrm{se}} \cos \Phi_{\mathrm{se}}=-\mathrm{z}_{\mathrm{se}} \tan \lambda$

or, $\Phi_{\mathrm{se}}=90.957464^{\circ}, 269.042536^{\circ}$

And for $\Phi_{\mathrm{se}}=90^{\circ}, \mathrm{x}_{\mathrm{se}}=0 \mathrm{~km}, \mathrm{x}_{\mathrm{so}}=2,499,787.40 \mathrm{~km}$., indicates that for orbital frame of reference, the center of the fictitious Celestial Sphere in ecliptic frame is appeared to be shifted by a distance of $2,499,787.40 \mathrm{~km}$. i.e. for Orbital frame of reference, the center of the fictitious Celestial sphere appears to be located at the point $\mathrm{S}$ instead of $\mathrm{S}^{\prime}$ as shown in fig. 2 , below. In other word, it can be said that, the center of the Celestial sphere in ecliptic frame of reference is shifted by $z_{\mathrm{se}} \sin \lambda$ for $\lambda$ degree inclined plane.

In frame Es, the value of $z_{\mathrm{se}}=811,459,649 \mathrm{~km}$. is constant throughout $360^{\circ}$ of rotation, indicates that the projection of $\mathrm{C}_{\mathrm{E}}$ " on ecliptic plane as $\mathrm{C}_{\mathrm{E}}{ }^{\prime}$, is on the equator of the Celestial Sphere.

At $\Phi_{\mathrm{se}}=90.478714^{\circ}$ and $269.521286^{\circ}$ of revolution, we have $\mathrm{z}_{\mathrm{se}}=\mathrm{z}_{\mathrm{so}}=811,459,649 \mathrm{~km}$, so that, the projection of $\mathrm{C}_{\mathrm{E}}$ " on orbital plane as $\mathrm{C}_{\mathrm{E}}$ lies at the equator of the Celestial Sphere. This means that, the orbital plane intersects the ecliptic plane at two points (as shown as point $\mathrm{E}$ in fig 2.); after $90.478714^{\circ}$ and $269.521286^{\circ}$ of revolution from the point where Earth is at aphelion.

The orbit of the Earth in orbital plane looks like elliptical with the Sun at one of its foci S (fig-3), with the semi-major axis 'a' equal to $149,597,870.7 \mathrm{~km}$. and the semi-minor axis 'b' equal to $149,576,983.3 \mathrm{~km}$. (which is equal to the value of $\mathrm{y}_{\mathrm{so}}$ at $\Phi_{\mathrm{se}}=90.957464^{\circ}$ and at this point $\left.\mathrm{x}_{\mathrm{so}}=0\right)$.

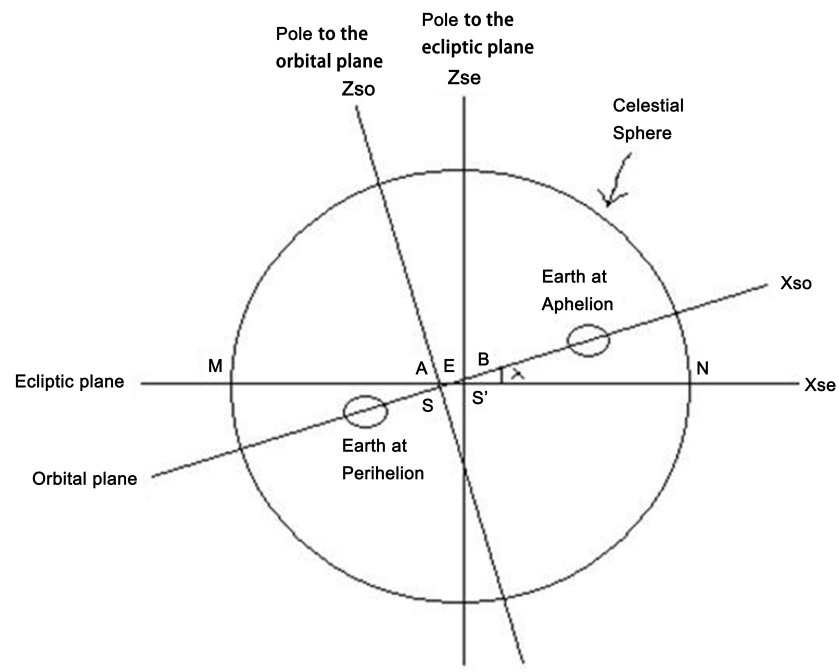

Figure 2. Celestial Sphere with Earth and Sun

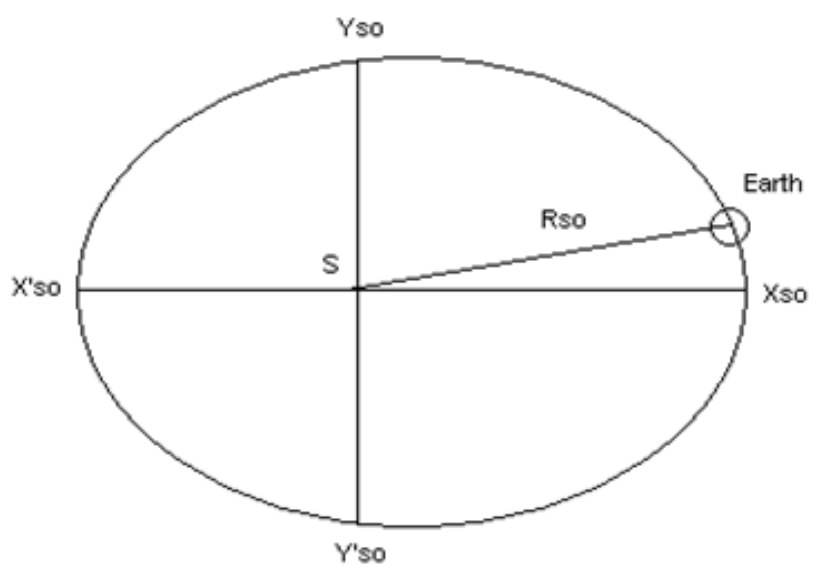

Figure 3. Orbital Plane of the Earth 


\subsection{Expression for the Obliquity of the Rotation Axis of Earth}

In Celestial Sphere, for any Sphere of radius $\mathrm{R}$, if the measurement is done along the center to the pole of ecliptic, then for $\eta$ degree inclined plane, the center of the Sphere is appeared to be shifted by the relation Rsinn.

In above case, the equatorial plane, or the equatorial circle of the fictitious Celestial Sphere is drawn at origin S'. For reverse case, if this Celestial Sphere is inclined by an angle $\eta$, then its center will be shifted to the point $\mathrm{S}$ and radial distance of $\mathrm{C}_{\mathrm{E}}$ ' from the center in ecliptic plane will be same as given in column 6 of table 1 . $\mathrm{km}$,

When $\Phi_{\mathrm{se}}=90^{\circ}, \mathrm{x}_{\mathrm{se}}=0 \mathrm{~km}, \mathrm{x}_{\mathrm{so}}=\mathrm{z}_{\mathrm{se}} \sin \lambda=2,499,787.4$

$$
\text { And } z_{\mathrm{so}}=z_{\mathrm{se}} \cos \lambda=811,455,798.6 \mathrm{~km} \text {. }
$$

i.e. for $\lambda$ degree inclined plane the value of $z_{s o}$ should be $\mathrm{z}_{\mathrm{se}} \cos \lambda$.

The value of $z_{\mathrm{se}}$ represents the distance between $\mathrm{C}_{\mathrm{E}}$ " and the center of the Earth of radius $6371 \mathrm{~km}$ in ecliptic plane and the Celestial Sphere is symmetric about ecliptic frame of reference.

$$
\begin{gathered}
\mathrm{C}_{\mathrm{E}}{ }^{\prime \prime} \mathrm{C}_{\mathrm{E}}^{\prime}-\mathrm{C}_{\mathrm{E}}{ }^{\prime \prime} \mathrm{C}_{\mathrm{E}}=\mathrm{z}_{\mathrm{se}}-\mathrm{z}_{\mathrm{So}} \\
=811,459,649 \mathrm{~km} .-811,455,798.6 \mathrm{~km} . \\
=3850.4 \mathrm{~km} .
\end{gathered}
$$

i.e. at this point, the value of $z_{\mathrm{se}}$ is greater than $z_{\mathrm{so}}$ by 3850.4 $\mathrm{km}$. This difference in value is compensated by inclination of Earth's axis to the normal to the ecliptic plane. For Earth of radius $6371 \mathrm{~km}$., the length measured is the deficiency of $(6371-3850.4=) 2520.6 \mathrm{~km}$, that value is equal to $6371 \mathrm{sin}$ $23.305675^{\circ}=R_{E} \sin \eta \mathrm{E}$, so that the obliquity of rotation axis is $23.305675^{\circ}$. That is the rotation axis of the Earth is inclined at $23.305675^{\circ}$ to the pole of the ecliptic.

\subsection{Inclination of the Sun's Axis to the Plane of Earth's Orbit}

For ecliptic frame of reference, the position of the Sun is at $S^{\prime}$ with its equator running along the plane of the ecliptic. But for $\lambda$ degree inclined orbital plane, the center of the Sun is appeared to be shifted at $\mathrm{S}$, where the value of $\left(\mathrm{x}_{\mathrm{so}}, \mathrm{y}_{\mathrm{so}}, \mathrm{z}_{\mathrm{so}}\right)$ for the position of Earth is

$$
\begin{gathered}
\mathrm{x}_{\mathrm{so}}=0 \mathrm{~km} ; \mathrm{y}_{\mathrm{so}}=149,576,983.3 \mathrm{~km} \text { and } \mathrm{z}_{\mathrm{so}}= \\
=811,463,499.5 \mathrm{~km} .=\mathrm{z}_{\mathrm{se}} / \cos \lambda
\end{gathered}
$$

The position of the Sun and the Earth become parallel to each other or lie in the same line for $\Phi_{\text {se }}=90.957464^{\circ}$ and $269.042536^{\circ}$ of revolution of Earth from the point where Earth is at aphelion distance. So the equinoxes occur at this point where $\Phi_{\mathrm{se}}=90.957464^{\circ}$ and $269.042536^{\circ}$.

At this position the value of $z_{\mathrm{so}}$ for Earth is related to $z_{\mathrm{se}}$ by the relation

$$
\mathrm{z}_{\mathrm{so}}=\mathrm{z}_{\mathrm{se}} / \cos \lambda
$$

Analogous to Earth, for the position of the Sun with center at $S^{\prime}$, the value of $z_{\mathrm{se}}$ is equal to the radius of the fictitious
Celestial sphere $\mathrm{R}_{\mathrm{c}}(=825,134,101.1 \mathrm{~km}$.) including the radius of the Sun. And for $\lambda$ degree inclined orbital plane, its value becomes $R_{c} / \cos \lambda$, same as in $\mathrm{eq}^{\mathrm{n}}$ (4), which is numerically equal to $825,138,016.4 \mathrm{~km}$.

The difference in value of $\mathrm{R}_{\mathrm{c}} / \cos \lambda$ and $\mathrm{R}_{\mathrm{c}}$ is $3,915.3 \mathrm{~km}$. and this difference in value for the Sun is compensated by inclination of Sun's axis to the plane of the Earth's orbit.

In case of Sun, radius of the Sun is

$$
\mathrm{R}_{\mathrm{s}}=695,990 \mathrm{~km} \text {. }
$$

And for $\lambda^{\circ}$ inclined Earth's orbital plane

$$
\begin{aligned}
\mathrm{R}_{\mathrm{s}} / \cos \eta_{\mathrm{s}} & =695,990 \mathrm{~km} .+3915.3 \mathrm{~km} . \\
& =699,905.3 \mathrm{~km} .
\end{aligned}
$$

Where, $\eta_{\mathrm{s}}$ is the inclination of Sun's axis to the plane of Earth's orbit.

or, $\cos \eta_{\mathrm{s}}=\mathrm{R}_{\mathrm{s}} / 699,905.3 \mathrm{~km}$.

or, $\eta_{\mathrm{s}}=6.063218^{\circ}$

i.e. for $\lambda=0.176506^{\circ}$ of inclined Earth's orbital plane, the inclination of Sun's axis to the plane of Earth's orbit is $\eta_{\mathrm{s}}=$ $6.063218^{\circ}$.

\subsection{Expression for the Variation in the Length of Solar Day throughout the Year}

Let us consider three frames of references: Earth's orbital frame of reference $\mathrm{O}_{\mathrm{E}}$, Earth's ecliptic frame of reference $\mathrm{E}_{\mathrm{E}}$ and Earth's rotational frame of reference $R_{E}$ fixed on Earth. Earth's orbital plane is a plane parallel to the orbital plane of the Celestial sphere, where the Sun's normal rays incident and complete a $360^{\circ}$ of revolution throughout the year. Earth's ecliptic plane is the plane parallel to the ecliptic plane of the Celestial sphere and the third plane is the Earth's rotational plane, in which the axis of Earth's rotation is tilted to the pole to the ecliptic by an angle of $\eta_{\mathrm{E}}\left(=23.305675^{\circ}\right)$, where all the astronomical event is observed.

Let the Cartesian coordinates of the Earth's orbital frame of reference $\mathrm{O}_{\mathrm{E}}$ is same as that in orbital frame of reference Os and is $\mathrm{x}_{\mathrm{so}}, \mathrm{y}_{\mathrm{so}}$ and $\mathrm{z}_{\mathrm{so}}$. The Cartesian coordinates of the Earth's ecliptic frame of reference $\mathrm{E}_{\mathrm{E}}$ is $\mathrm{x}_{\mathrm{Ee}}, \mathrm{y}_{\mathrm{Ee}}$ and $\mathrm{z}_{\mathrm{Ee}}$; and that of the Earth's rotational frame of reference $\mathrm{R}_{\mathrm{E}}$ are $\mathrm{x}_{\mathrm{Er}}, \mathrm{y}_{\mathrm{Er}}$ and $\mathrm{z}_{\mathrm{Er}}$ respectively.

Let $\Phi_{\mathrm{so}}, \Phi_{\mathrm{Ee}}$ and $\Phi_{\mathrm{Er}}$ are the longitudinal or azimuthal angle in $\mathrm{O}_{\mathrm{E}}, \mathrm{E}_{\mathrm{E}}$ and $\mathrm{R}_{\mathrm{E}}$ respectively. Where

$$
\tan \Phi_{\mathrm{so}}=\mathrm{y}_{\mathrm{so}} / \mathrm{x}_{\mathrm{so}} ; \tan \Phi_{\mathrm{Ee}}=\mathrm{y}_{\mathrm{Ee}} / \mathrm{x}_{\mathrm{Ee}} \text { and } \tan \Phi_{\mathrm{Er}}=\mathrm{y}_{\mathrm{Er}} / \mathrm{x}_{\mathrm{Er}}
$$

Let $\mathrm{d} \Phi_{\mathrm{so}}{ }^{\circ}$ represents the degree of revolution of Earth on the orbital plane of the fictitious Celestial sphere as well as the motion of the Sun's normal rays on the Earth's orbital plane per solar day. In the same way, $\mathrm{d} \Phi_{\mathrm{Ee}}{ }^{\circ}$ and $\mathrm{d} \Phi_{\mathrm{Er}}{ }^{\circ}$ represent the corresponding value of $\mathrm{d} \Phi_{\mathrm{so}}{ }^{\circ}$ in Ecliptic plane and rotational plane of the Earth's surface per solar day respectively.

Since the value of $\Phi_{\mathrm{so}}$ and $\Phi_{\mathrm{Ee}}$ both lie on the surface of the Earth so to change the value of $\Phi_{\mathrm{so}}$ to $\Phi_{\mathrm{Ee}}$ in case of Earth, the value of $\lambda$ is replaced by $\theta_{\mathrm{E}}$, which is the angle subtended between $R_{\text {se }}$ and $R_{\text {so }}$, and as like the value of $\lambda$, the value of $\theta_{E}$ 
also varies from $+\theta_{\mathrm{E}}$ to $0^{\circ}$ and then from $0^{\circ}$ to $-\theta_{\mathrm{E}}$.

To convert the value $\Phi_{\mathrm{Ee}}$ in Earth's ecliptic plane to Earth's rotational plane $\left(\Phi_{\mathrm{Er}}\right)$, we have the relations,

$$
\begin{array}{r}
\tan \Phi_{\mathrm{Er}}=\left(\tan \Phi_{\mathrm{so}} / \cos \eta_{\mathrm{E}^{\mathrm{o}}}\right) \times \cos \theta_{\mathrm{E}}, \text { for } \Phi_{\mathrm{se}}<90.478714^{\circ} \text { and } \\
\Phi_{\mathrm{se}}>269.521286^{\circ} \\
=\tan \Phi_{\mathrm{so}} / \cos \eta \mathrm{E}^{\mathrm{o}}, \text { for } \Phi_{\mathrm{se}}=90.478714^{\circ}=269.521286^{\circ}(5 \mathrm{~B}) \\
=\tan \Phi_{\mathrm{so}} /\left(\cos \theta_{\mathrm{E}} \times \cos \eta \mathrm{E}^{\circ}\right), \text { for } \Phi_{\mathrm{se}}>90.478714^{\circ} \text { and } \\
\Phi_{\mathrm{se}}<269.521286^{\circ}
\end{array}
$$

Earth revolves around the Sun at the same time it also rotates on its axis. Solar day is the length of the time between one local noon to the next and the length of the solar day is not always 24 hours and its average over the course of a year is 24 hours or 86,400 seconds.

An apparent solar day is the amount of time elapsed between two passages of the Sun at the meridian and the length of the apparent solar day varies throughout the year. The length of the tropical year is $31,556,925.25$ seconds and one tropical year is 365.242190402 mean solar days.

\section{Case I: For Ecliptic Plane:}

Let $\mathrm{d} \Phi_{\text {se }}^{\prime}{ }^{\circ}, \mathrm{d} \Phi_{\mathrm{se}}{ }^{\circ}$ and $\mathrm{d} \Phi^{\prime \prime}{ }_{\text {se }}{ }^{\circ}$ represent the angle subtended by Earth in Ecliptic plane of the Celestial sphere in one sidereal day, one mean solar day and the one apparent solar day respectively. Then, according to the tropical year, the degree of advancement of Earth along its orbit for one sidereal day [3] $\left(=\mathrm{d} \Phi^{\prime}{ }_{\mathrm{se}}{ }^{\circ}\right)$, which is $360^{\circ}$ of rotation of Earth on its axis, is

$(86,164.09053 \mathrm{sec} / 31,556,925.25 \mathrm{sec}.) \times 360^{\circ}=0.982956^{\circ}$

And, the degree of advancement of Earth along its orbit for the Sun in one mean solar day $\left(=\mathrm{d} \Phi_{\mathrm{se}}{ }^{\circ}\right)$, which is more than $360^{\circ}$ of rotation of Earth on its axis, is

$$
(86,400 . \mathrm{sec} / 31,556,925.25 \mathrm{sec} .) \times 360^{\circ}=0.985647^{\circ}
$$

That means, $0.982956^{\circ}$ of revolution of Earth around the Sun $=360^{\circ}$ of rotation of Earth on its axis.

And $0.985647^{\circ}$ of revolution of Earth around the Sun

$=360.9856^{\circ}$ of rotation of Earth on its axis.

That is, between two successive noons at any point on Earth surface in the ecliptic plane of the fictitious Celestial sphere, Earth actually rotates through $360.9856^{\circ}$. For one solar day, the value of degree of advancement of Earth along its orbit and the extra degree of rotation of Earth on its axis (or more than $360^{\circ}$ of rotation) in order to face the same terrestrial meridian are same and is equal to $0.9856^{\circ}$. In another word, for $\Phi_{\mathrm{se}}{ }^{\circ}=0.985647^{\circ}$ of advancement of Earth around the Sun in the ecliptic plane of the Celestial sphere, the extra angle Earth must rotate on its axis to get the Sun back to the meridian is also $0.9856^{\circ}$.

In general, for $\mathrm{d} \Phi_{\mathrm{se}}{ }^{\circ}$ of revolution of Earth around the Sun, the position for next noon to occur is also changed by $\mathrm{d} \Phi_{\mathrm{se}}{ }^{\circ}$, so for this an additional turn $0.9856^{\circ}$ must be rotated by Earth in order to return to the same meridian relative to the Sun.

\section{Case II: For Orbital Plane:}

The value of $\mathrm{d} \Phi_{\mathrm{so}}{ }^{\circ}$ represents not only the degree of revolution of Earth around the Sun per solar day in orbital frame of reference, but also the degree of revolution of Sun's normal rays on the Earth's circumference in Earth's orbital plane, which is parallel to the orbital plane of the Celestial sphere.

In ecliptic frame of reference $E_{S}$, the value of the degree of revolution of Earth around the Sun per day is uniform and is equal to $0.982956^{\circ}\left(=\mathrm{d} \Phi_{\mathrm{se}}^{\prime}{ }^{\circ}\right)$ for one sidereal day and $0.985647^{\circ}\left(=\mathrm{d} \Phi_{\mathrm{se}}{ }^{\circ}\right)$ for one mean solar day, but the value of $\mathrm{d} \Phi_{\mathrm{so}}{ }^{\circ}$ in orbital frame of reference for the same interval of time is different in different location, during revolution of Earth around the Sun.

Now on doing calculations by using eq ${ }^{\mathrm{n}}$ (5), for $1^{\text {st }}$ day of the aphelion, it is found that the length of the apparent solar day is longer than the mean solar day by $12.56606 \mathrm{sec}$.

Similarly, in $1^{\text {st }}$ day of the perihelion, the length of the apparent day is calculated to be longer than the mean solar day by $29.81240 \mathrm{sec}$ or nearly 30 seconds.

In this way, length of the apparent solar day of any particular day can be calculated by mathematical formula. Thus, the length of the solar day can be calculated mathematically throughout the year. The difference in mean solar day and apparent solar day arise mainly due to the motion of Earth in an inclined orbital plane and the obliquity of the rotational axis of the Earth, i.e. the length of the apparent solar day is observed in rotational plane of the Earth.

The mathematically derived value of the length of the apparent solar day matches with the observed result (e.g. Giesen [4]). This result indicates that Earth rotates and revolves around the Sun with uniform velocity.

\subsection{Expression for the Rate of Precession}

Though Earth revolves and rotates around the Sun with uniform velocity, it appears that it moves faster at perihelion than at the aphelion. In the same way, the difference in time period between sidereal year and tropical year of $1,224.48$ sec is observed in the rotational plane of the Earth, which is inclined at an angle of $\mathrm{\eta}^{\mathrm{o}}$, with the ecliptic plane. That is, the rate of precession is $0.013968^{\circ}\left(=\Phi_{\mathrm{Er}}{ }^{\circ}\right)$ or 50.288400 arc seconds per year, observed in Earth's rotational plane. Let Sun revolves around the Galactic center in ecliptic plane, then to calculate the value of precession of $0.013969^{\circ}$ or 50.2884 " arc seconds $\left(=\Phi_{\mathrm{Er}}\right)$ in ecliptic plane of the galaxy as $\Phi_{\mathrm{GC}}$ we have the relation,

$$
\begin{gathered}
\tan \Phi_{\mathrm{GC}}=\tan \Phi_{\mathrm{Er}} / \cos \eta \mathrm{E}^{\circ} \\
=\tan 0.013969^{\circ} / \cos 23.305675^{\circ}
\end{gathered}
$$

or $\Phi_{\mathrm{GC}}=0.015210^{\circ}$ or 54.75610 seconds of arc per year.

That is the rate of precession of Earth in Earth's rotational plane is found to be 50.2858 seconds of arc but in ecliptic plane is 54.75610 seconds of arc. In another word, the Sun's yearly shift from $S$ ' to $S$ (relative to the background stars), causes the position of the stars changes by 50.28 arc seconds 
every year in Earth's rotational frame of reference.

\subsection{Saurya Day}

In modern astronomy, the zodiac is divided into twelve signs, viz, Aries, Tarus, Gemini, Cancer, Leo, Virgo, Libra, Scorpio, Sagittarius, Capricorn, Aquarius and Pisces, starting from the vernal equinoctial point called "the first point of Aries". The celestial longitudes of heavenly bodies measured along such a moving zodiac are referred to as Tropical or sayana longitudes [5].

In Indian astronomy, the celestial longitudes are measured starting from a fixed point of reference called sidereal or nirayana or Mesadi longitudes. In Indian astronomy, the zodiac is divided into twelve signs viz, Mesa, Vrsabha, Mithuna, Karkataka, Simha, Kanya, Tula, Vrscika, Dhanus, Makara, Kumbha and Mina [5].

The first point of Mesa is the starting point of the zodiac in the Indian system. The first point of Aries moves backward constantly as compared to the first point of Mesa due to precession of the equinoxes. In fig.2, initially the position of the Sun and stars is at $S^{\prime}$ and after one year, the position of the Sun changed from $S$ ' to $S$ for $\lambda^{\circ}$ inclined orbital plane, so that Earth has to rotate $1224.48 \mathrm{sec}$ more for the fixed stars.

Consider an Orbital-Circle of the Earth, which is divided into twelve signs and where the first point of Mesa coincided with the first point of Aries as shown in fig.4.

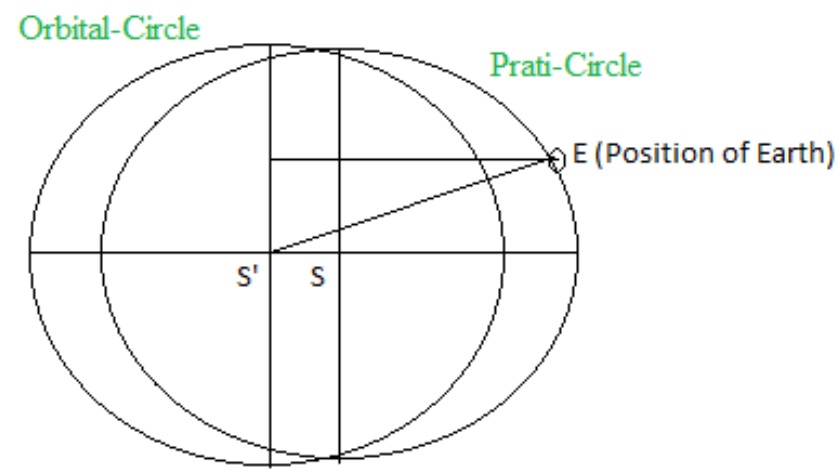

Figure 4. Seperation of the Prati-Circle from the Orbital-Circle

For the planet Earth, the Sun together with the Earth moves away from the fixed zodiac along $\mathrm{xx}$ '-axis at the rate of $0.0792 \mathrm{~km} / \mathrm{sec}$. ( = 2,499,798.6 km. $/ 31,558,149.73 \mathrm{sec})$, causes the motion of the Earth with Sun in a Prati-Circle (In Sanskrit, it is called Prativrtta in Surya-Siddhanta [6]) as shown in fig.4. In another word Prati-Circle is moving away from the Orbital-Circle at the rate of $0.0792 \mathrm{~km} / \mathrm{sec}$. as shown in Fig. 4

When Earth turns one rotation on its axis w. r. t. the fixed stars, the Sun has separated from the fixed stars along $x x^{\prime}$-axis at the rate of $0.0792 \mathrm{~km} / \mathrm{sec}$. Now taking on account of motion of Sun from S' towards S, on the circumference where Earth revolves around the Sun, Earth has to rotate $1497.43 \mathrm{sec}$. more to compensate this length with respect to the Sun, so that the length of the day must be of $87661.52 \mathrm{sec}$.
In another word, like sidereal day $(86,164.09053 \mathrm{sec})$, solar day $(86400 \mathrm{sec})$, let us introduce another length of the day called Saurya day (Sanskrit word given in Surya-Siddhanta [6]) of length $87661.527 \mathrm{sec}$. so that in one sidereal year, there is 360 Saurya days of length 87661.527 sec. (= $86164.090531 \mathrm{sec}+1497.43 \mathrm{sec})$.

The length of the Saurya day arises mainly due to the motion of the Prati-Circle, which is moving away from the orbital-Circle. In one Saurya day, the time period of 1497.43 sec arises due to the two factors: angular and linear separation of stars from the Earth. Due to these two reasons, it is found mathematically that the time period for the motion of Sun from S' towards S and the motion of Earth on the circumference of the orbital plane is related to each other by the factor of 0.3. And in each Saurya day, the angular separation of the stars (for a moment neglecting linear separation) with the Earth is $6^{\circ}$.

For Earth's rotation on its axis, SS' represents the time interval of 1224.48 secs, whereas for Earth's revolution, SS' represents the separation between the position of the stars and Sun in one year and from fig 2, this distance is equal to $2,499,798.6 \mathrm{~km}$. Earth revolves around the Sun at the same time within solar system it travels around the Galactic center.

This value in Earth's rotational plane is 50.2858 seconds of arc and in ecliptic plane is 54.7532 seconds of arc. In this case also, if the linear separation of the stars is neglected, then the angular distance between the fixed stars and Sun is found to be 54" of arcs. But due to the factor of 0.3, the vernal equinox must have a precession rate of about 180 " $(=54 \% / 0.3)$ of arc per year in ecliptic plane. So on ecliptic plane, vernal equinox may precesses slowly with respect to the fixed stars, completing one revolution in about 7,200 years.

Due to the factor of 0.3, an Earth bound observer who observes the entire celestial phenomenon in Earth's rotational plane, may see the $90^{\circ}$ of precession of equinox at $27^{\circ}$. That is the vernal equinox point appears to be rises from $0^{\circ}$ to $27^{\circ}$ during 1800 years, then decreases to $0^{\circ}$ and further to $-27^{\circ}$, there after rising again, thus oscillating within a range of $\pm 27^{\circ}$, so that cyclically motion of the precession of the equinox may appears as oscillating within a range of \pm $27^{\circ}[7]$.

The time taken for the Sun to move from the vernal equinox to the summer solstice, summer solstice to the autumnal equinox, autumnal equinox to the winter solstice and then back to the vernal equinox are 93.00045, 93.91475, 90.097384 and 89.23252 sidereal days respectively [8].

As given in section 3.4, for $\lambda^{\circ}$ inclined orbital plane, the center of the fictitious Celestial sphere is shifted at the $\Phi_{\mathrm{se}}=$ $90.957464^{\circ}$ from the point of aphelion distance, so that equinox must occurs at this point. Taking the consideration of the motion of Prati-Circle away from the Orbital-Circle (fig.4) also, mathematically the time taken for the Sun to move from the vernal equinox to the summer solstice is 93.0077 sidereal days is derived. In the same way, the time taken for the Sun to move from summer solstice to autumnal 
equinox, autumnal equinox to the winter solstice and back to the vernal equinox are 93.9177, 90.0913 and 89.2338 sidereal days respectively are derived.

\section{Results}

a) The deviation in the path of the Earth from the circle indicates that the Earth's orbital plane is inclined at an angle of 0.176506 degree with the ecliptic plane.

b) The distance between the Earth and the Sun $\left(\mathrm{R}_{\mathrm{so}}\right)$ at any instant in orbital plane is obtained by using the formula, $\mathrm{R}_{\mathrm{so}}=\left(\mathrm{x}_{\mathrm{so}}{ }^{2}+\mathrm{y}_{\mathrm{so}}{ }^{2}\right)^{1 / 2}$ in $360^{\circ}$ revolution period. Here, $360^{\circ}$ period of revolution indicates the total number of days 365.242190 in one tropical year.

c) The obliquity of the rotation axis of the Earth and the Inclination of the Sun's axis to the plane of the Earth's orbit is mathematically found.

d) The variation in the length of the solar day can be found by the mathematical formula.

e) Due to the motion of the Prati-Circle away from the Orbital-Circle, new type of the length of the day called Saurya day $(=87,661.527 \mathrm{sec}$. $)$ is introduced, so that the rate of precession is calculated on this basis.

f) On the basis of the length of the Saurya day, the time period for the Sun to move from the vernal equinox to summer solstice, to the autumnal equinox to the winter solstice and back to the vernal equinox are derived mathematically.

\section{Conclusions}

Thus the conclusions obtained from above analysis are that

1) The orbit of a planet is in an inclined plane of spherically symmetric system, which appears elliptical with the Sun at one focus.

2) The planet rotates and revolves around the Sun with uniform velocity.
3) The variation in the length of the solar day arises due to the motion of Earth in an orbital plane and inclination of the Earth's axis relative to the Earth's ecliptic plane.

4) For an inclined orbital plane, Earth revolves around Sun in a Prati-Circle and at the same time, moves away from the Orbital-Circle, are the main cause for the phenomenon of precession of equinox.

\section{Acknowledgements}

We would like to express our gratitude to the book "Plant and Planet Earth" by Mahendra Lall Karna, 2003.

\section{REFERENCES}

[1] Emiliani C. Planet, Earth, Cambridge University Press; 1992.p. 160-1.

[2] Mathur DS. Mechanics, S. Chand \& Company Ltd. 818, 1996.p. 77-2.

[3] Sidereal time. [cited 2010 Sep 6]. Available from: https://en.wikipedia.org/wiki/Sidereal_time

[4] Giesen J. Equation of time and length of solar day. [cited 2016 Nov 6]. Available from: http://www.jgiesen.de/Divers/Sonne nuhr/eot.html

[5] Balachandra Rao S. Indian astronomy: an introduction. Hyderabad, AP: University Press; 2002.

[6] Pt. Madhava P. Purohit, Surya-Siddhanta, Chaukhamba Surbhaarati Prakashan; 2000

[7] Ayanamsa. [cited 2016 August 4]. Available from: http://en.wikipedia.org/wiki/Ayanamsa

[8] Equinox. [cited 2012 Feb 15]. Available from: http://en.wikipedia.org/wiki/Equinox 